\title{
SNX-2112 Induces Apoptosis and Autophagy of Nara-H Cells
}

\author{
NATSUKO FUKUOKA, OSAMU NAKAMURA, YOSHIKI YAMAGAMI, \\ HIDEKI NISHIMURA, YOICHI ISHIBASHI and TETSUJI YAMAMOTO
}

\author{
Department of Orthopaedic Surgery, Kagawa University Faculty of Medicine, Kagawa, Japan
}

\begin{abstract}
Background/Aim: Selective heat shock protein 90 (Hsp90) inhibitor SNX-2112 exhibits antitumor activity in multiple cancer cell types. Here, the antitumor activity of SNX2112 in Nara-H cells was analyzed. Materials and Methods: Antitumor activity of SNX-2112 was assessed using a cell proliferation assay. We also examined the signalling pathways involved in SNX-2112-mediated autophagy and apoptosis of Nara-H cells by western blot and morphological analyses. Results: Cell proliferation assays demonstrated that SNX-2112 inhibited Nara-H cell growth. Western blotting revealed that SNX-2112 induced apoptosis and autophagy, inhibited mammalian target of rapamycin (mTOR) phosphorylation, and suppressed the mitogen-activated protein kinase (MAPK) signalling pathway. Morphological analysis confirmed that SNX-2112 induced autophagy and apoptosis. Conclusion: SNX2112 induced autophagy and apoptosis of Nara-H cells by inhibiting mTOR and MAPK pathways. Our results support developing SNX-2112 to treat human soft tissue sarcomas.
\end{abstract}

Undifferentiated pleomorphic sarcoma (UPS) is a heterogeneous tumour group without an identifiable lineage. Despite many chemotherapy protocols available for UPS, little consensus exists concerning appropriate doses and agent combinations $(1,2)$.

Heat shock proteins (Hsps) are potential targets for molecular targeted therapy. Cancer patients with high Hsp90 levels often have low survival rates. Thus, blocking Hsp90 may be a novel therapeutic strategy $(3,4)$, and the selective Hsp90 inhibitor SNX-2112 shows significant antitumor activity in several cancer cell types (5).

Autophagy has recently gained attention in regard to cancer pathogenesis and treatment, because of its paradoxical

Correspondence to: Osamu Nakamura, Department of Orthopaedic Surgery, Kagawa University Faculty of Medicine, 1750-1 Ikenobe, Miki-cho, Kita-gun, Kagawa 761-0793, Japan. Tel: +81 878912195, Fax: +81 878912196, e-mail: osamunak@ med.kagawa-u.ac.jp

Key Words: SNX-2112, autophagy, apoptosis, undifferentiated pleomorphic sarcoma. roles in cell survival and death (6). Hsp90 inhibition induces autophagy through suppression of the mammalian target of rapamycin (mTOR) (7). We previously demonstrated that an mTOR inhibitor induced cytoprotective autophagy in Nara-H cells, a high-grade UPS cell line (8). Autophagy protects cells against antitumor agents, and inhibiting autophagy enhances apoptosis (9-11). Here, the antitumor activity of SNX-2112 and the signalling pathways involved in SNX2112-mediated autophagy and apoptosis were assessed in Nara-H cells.

\section{Materials and Methods}

SNX-2112 and Nara-H cell culture. SNX-2112 was purchased from Selleckchem (Houston, TX, USA). Nara-H cells were obtained from ScienStuff (Nara, Japan) and cultured at $37^{\circ} \mathrm{C}$ with $5 \% \mathrm{CO}_{2}$.

Cell proliferation assay. The CellTiter $96^{\circledR}$ AQueous One Solution Cell Proliferation Assay (Promega, Madison, WI, USA) was used to analyze cellular proliferation. Cells were seeded at $1 \times 10^{4}$ cells/well in 96-well plates. Forty-eight $\mathrm{h}$ later, fresh medium with $0,0.4,2,10$, or $50 \mu \mathrm{M}$ SNX-2112 was added. After 24 or 48 h, 20 $\mu l$ of 3-(4,5-dimethylthiazol-2-yl)-5-(3-carboxymethoxyphenyl)2-(4sulfophenyl)-2H-tetrazolium in $100 \mu$ of fresh medium was added to each well. The cultures were incubated for $2 \mathrm{~h}$, and then optical density was determined at $490 \mathrm{~nm}$ using an automatic microplate reader (Molecular Devices, Sunnyvale, CA, USA). At least three independent experiments were performed for each condition.

Western blotting. Cells were seeded at $\sim 6 \times 10^{5}$ cells/well in six-well plates and cultured for $48 \mathrm{~h}$. Cells were treated with SNX-2112 for $24 \mathrm{~h}$ and lysed in lysis buffer (Cell Signaling Technology, Beverly, MA, USA). Equivalent protein amounts were separated by SDSpolyacrylamide gel electrophoresis (Wako, Tokyo, Japan) under reducing conditions, electrophoretically transferred to nitrocellulose membranes (GE Healthcare Bio-Sciences, Piscataway, NJ, USA), and sequentially incubated with primary and secondary antibodies (Table I) in iBind solution (iBind Western System, Life Technologies, Carlsbad, CA, USA) for $2.5 \mathrm{~h}$. Labelled proteins were visualized with a Novex ${ }^{\circledR}$ AP Chemiluminescent Detection Kit (Life Technologies) and LAS-1000 plus image analyzer (Fujifilm Co., Tokyo, Japan).

Immunocytochemistry of microtubule-associated protein light-chain 3 (LC3). Cells were plated at $1 \times 10^{6}$ cells/well on $25-\mathrm{mm}$ round 
Table I. Primary antibodies used in western blot analysis.

\begin{tabular}{lcccc}
\hline Target & Source & Host & Dilution & Secondary antibody \\
\hline HSP90 & Cell Signaling & Rabbit & $1: 1,000$ & Anti-rabbit \\
Phospho-HSP90 $\alpha$ & Cell Signaling & Rabbit & $1: 1,000$ & Anti-rabbit \\
4E-BP1 & Cell Signaling & Rabbit & $1: 1,000$ & Anti-rabbit \\
Phospho-4E-BP1 & Cell Signaling & Rabbit & $1: 1,000$ & Anti-rabbit \\
p70S6K & Cell Signaling & Rabbit & $1: 1,000$ & Anti-rabbit \\
Phospho-p70S6K & Cell Signaling & Rabbit & $1: 1,000$ & Anti-rabbit \\
MEK1/2 & Cell Signaling & Rabbit & $1: 1,000$ & Anti-rabbit \\
Phospho-MEK1/2 & Cell Signaling & Rabbit & $1: 1,000$ & Anti-rabbit \\
ERK1/2 & Cell Signaling & Rabbit & $1: 1,000$ & Anti-rabbit \\
Phospho-ERK1/2 & Chemicon & Rabbit & $1: 1,000$ & Anti-rabbit \\
B-Raf & Cell Signaling & Rabbit & $1: 1,000$ & Anti-rabbit \\
Cleaved Caspase-9 & Cell Signaling & Rabbit & $1: 1,000$ & Anti-rabbit \\
Cleaved PARP & Cell Signaling & Rabbit & $1: 1,000$ & Anti-rabbit \\
$\alpha$-tubulin & Cell Signaling & Rabbit & $1: 1,000$ & Anti-rabbit \\
Akt & Sigma & Rabbit & $1: 1,000$ & Anti-rabbit \\
Phospho-Akt & Sigma & Rabbit & $1: 1,000$ & 10 \\
LC-3 & MBL & Rabbit & $1: 1,000$ & Anti-rabbit \\
Atg5-Atg12 complex & MBL & Rabbit & $1: 1,000$ & Anti-rabbit \\
p62/SQSTM1 & MBL & Rabbit & $1: 1,000$ & Anti-rabbit \\
\hline
\end{tabular}

coverslips (Matsunami Glass Ind. Ltd., Osaka, Japan) and cultured overnight. Cells were then treated with $25 \mu \mathrm{M}$ SNX-2112 for $24 \mathrm{~h}$, fixed in 4\% paraformaldehyde/phosphate-buffered saline (PBS) for $30 \mathrm{~min}$ at room temperature, and then rinsed with PBS. To detect autophagy, fixed cells were stained with an anti-LC3 antibody (Code No. PM036; MBL, Nagoya, Japan) for $1 \mathrm{~h}$ at room temperature. After two washes, cells were incubated with an antiIgG secondary antibody (Alexa Fluor ${ }^{\circledR}$ 488, Code No. A11008; Invitrogen, Carlsbad, CA, USA) for $30 \mathrm{~min}$ at room temperature and then viewed under an epifluorescence microscope (FSX100; Olympus Optical Co., Ltd., Tokyo, Japan).

Apoptosis detection by morphological assessment. Cells were plated and incubated with $25 \mu \mathrm{M}$ SNX-2112 as described above. The cells were then stained with annexin V-FITC, propidium iodide (PI), and Hoechst 33342 for $15 \mathrm{~min}$ in the dark using reagents in the Promokine Apoptotic/Necrotic/Healthy Cells Detection Kit (PromoCell GmbH, Heidelberg, Germany).

Transmission electron microscopy. Cells were plated at $1 \times 10^{4}$ cells/well on a Lab-Tek ${ }^{\circledR}$ Chamber Slide (Nalge Nunc International, Naperville, IL, USA) and cultured for $48 \mathrm{~h}$. After treatment with 25 $\mu \mathrm{M}$ of SNX-2112 for $24 \mathrm{~h}$, the cells were rinsed, fixed with $2.5 \%$ glutaraldehyde in $0.1 \mathrm{M}$ phosphate buffer ( $\mathrm{pH} 7.4$ ) for $2 \mathrm{~h}$, and postfixed in $1 \%$ osmium tetroxide in the same buffer for $2 \mathrm{~h}$. Ultrathin sections were stained with $4 \%$ uranyl acetate and lead citrate, and viewed under a JEM-1400 electron microscope ( $80 \mathrm{kV}$ accelerating voltage; Jeol, Tokyo, Japan).

Statistical analysis. Proliferation assay results were assessed with one- or two-way analysis of variance, followed by post-hoc analysis. $p<0.05$ was considered as statistically significant. Statistical analyses were performed using GraphPad Prism 5 software (GraphPad, San Diego, CA, USA).

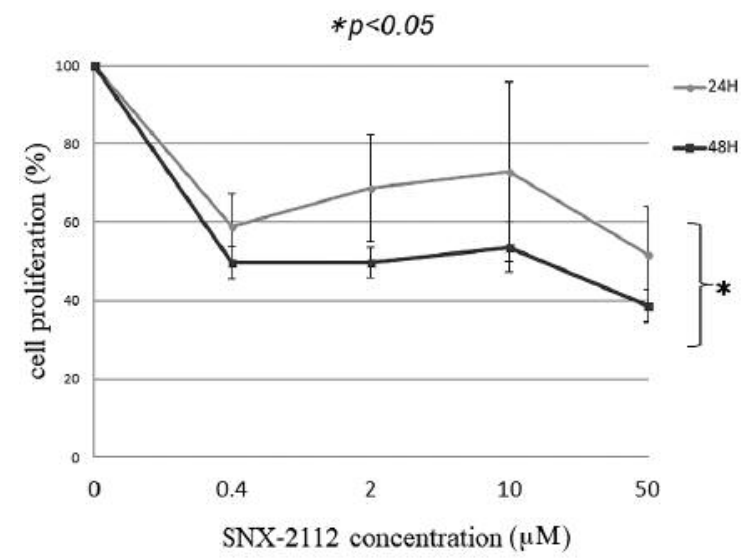

Figure 1. SNX-2112 inhibits Nara-H cell proliferation.

\section{Results}

SNX-2112 inhibits cell proliferation. SNX-2112 inhibited Nara-H cell proliferation in a dose and time-dependent manner (Figure 1).

Western blot analysis demonstrated that SNX-2112 induced apoptosis and autophagy, and inhibited mTOR and MAPK signalling pathways. HSP90 expression was high in Nara-H cells, and SNX-2112 decreased p-HSP90 $\alpha$ expression in a dose-dependent manner (Figure 2A). SNX-2112 also induced dose and time-dependent down-regulation of p-p70S6K and p-4E-BP1, which are downstream of the Akt/mTOR 

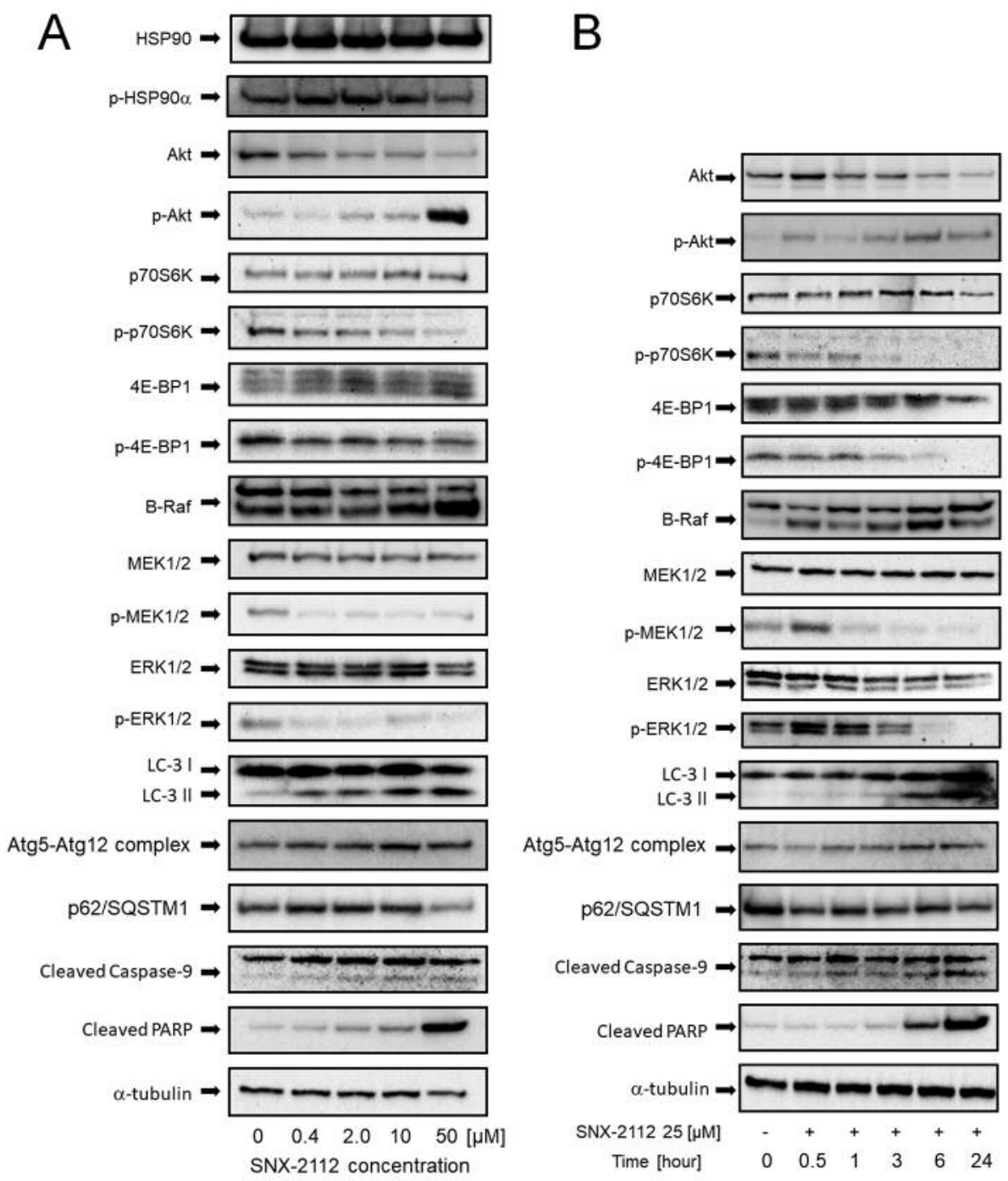

Figure 2. Western blot analysis of SNX-2112 effects. (A) SNX-2112 induced dose-dependent down-regulation of mTOR and MAPK pathways, and apoptosis and autophagy were induced in Nara-H cells treated with SNX-2112. (B) Time-dependent effects of SNX-2112 in Nara-H cells.

signalling pathway, and up-regulation of p-Akt (an activator of the Akt/mTOR signalling pathway) in Nara-H cells (Figure $2 \mathrm{~A}$ and $\mathrm{B}$ ).

In regard to $\mathrm{B}-\mathrm{Raf} / \mathrm{mitogen}$-activated protein kinase (MAPK) signalling, treatment with SNX-2112 reduced pMEK1/2 and p-ERK1/2 expression, while B-Raf expression was up-regulated in a dose and time-dependent manner (Figure 2A and B).

SNX-2112 treatment led to Poly (ADP-ribose) polymerase (PARP) cleavage and activation of caspase-9 in a dose and time-dependent manner in Nara-H cells, suggesting that SNX-2112 induced apoptosis (Figure 2A and B). Inhibition of PARP cleavage allows cells to maintain viability, whereas PARP cleavage facilitates cellular disassembly and serves as a marker of cells undergoing apoptosis (12).
SNX-2112 treatment increased expression of autophagyrelated proteins LC3-II and Atg5-Atg12 complex. p62/SQSTM1 expression was decreased in a dose and timedependent manner, indicating that SNX-2112 also induced autophagy of Nara-H cells (Figure 2A and B).

Morphological analysis revealed that SNX-2112 increased LC3positive cells and apoptotic cells. LC3-positive cells were increased among SNX-2112-treated Nara-H cells compared with control cells (Figure 3A). In addition, in the presence of SNX2112 annexin V-FITC-positive and annexin V-FITC/PI doublepositive cells were identified, indicating apoptosis (Figure 3B).

Electron microscopy demonstrated that there were autophagic cells and apoptotic cells in SNX-2112 treated cells. Using 


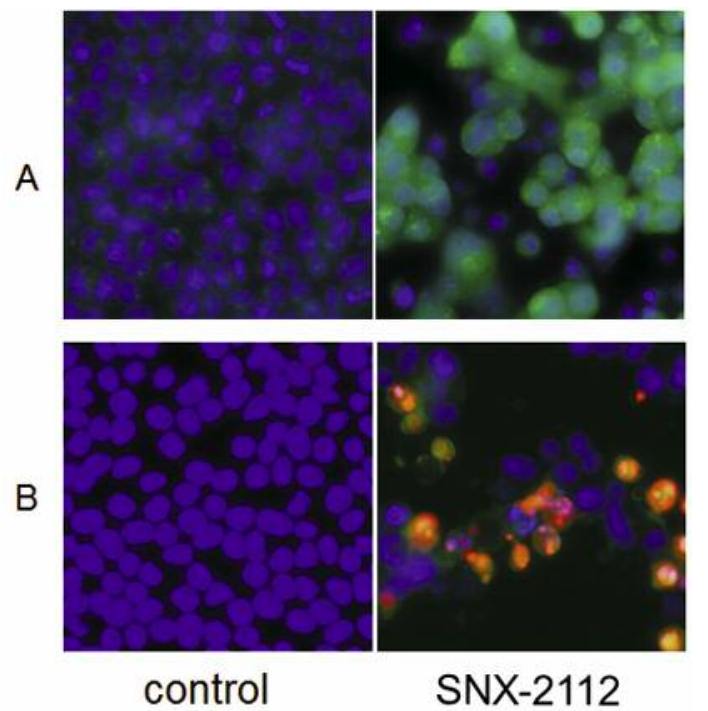

Figure 3. Morphological analysis by fluorescence microscopy. (A) LC3 was used to evaluate autophagy. (B) Apoptotic cells were detected by annexin V, PI, and Hoechst 33342 triple staining.

electron microscopy SNX-2112-treated cells were found to contain autophagosomes (Figure 4A and B). Apoptotic cells with nuclear fragmentation and chromatin condensation were also identified (Figure 4C).

\section{Discussion}

Molecular targeted drugs, including mTOR inhibitors, are being tested for sarcoma as monotherapy or in combination with other drugs such as autophagy inhibitors $(13,14)$. Because Hsp90 interacts with proteins in multiple signalling pathways necessary for cancer cell survival, such as mTOR and MAPK pathways, Hsp90 inhibitors have attracted great interest for cancer treatment. Hsp90 is constitutively expressed at 2-10-fold higher levels in tumour cells compared with normal cells, suggesting that Hsp90 may play a crucial role in the growth and survival of tumour cells (15). Moreover, Hsp90 interacting proteins induce apoptosis in various tumours (16). Therefore, Hsp90 inhibition is attractive as a therapeutic strategy for cancer.

Hsp90 blockage in cancer cells leads to protein breakdown and possibly lower proliferation. Clinical trials are testing Hsp90 inhibitors alone or in combination with other drugs as a therapy for multiple cancers (16). SNX-2112 inhibits, both in vitro and in vivo, growth of multiple cancer cell lines $(17,18)$. However, little is known regarding the activity of SNX-2112 in soft tissue tumours such as UPS. Thus, the antitumor effects of SNX-2112 were investigated in Nara-H cells. Consistent with observations in other cancers, SNX-2112 effectively inhibited Nara-H cell proliferation in a dose and time-dependent manner.

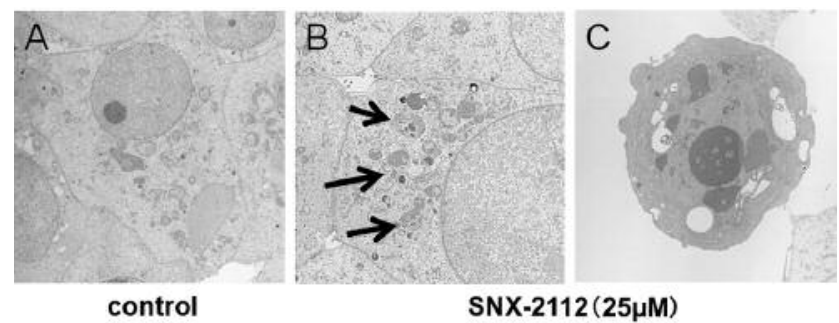

Figure 4. Electron microscopy. (A) Control cell. (B) Autophagosomes (arrows). (C) Apoptotic cell.

mTOR modulates several cell signalling events in the PI3K/Akt pathway that is important for the proliferation of many cancer cell types (14). mTOR interacts with other proteins and is the core component of two biochemically distinct complexes called mTORC1 and mTORC2. mTORC1, but not mTORC2, regulates autophagy and cell growth, the latter via p70S6K and 4E-BP1 phosphorylation (19, 20). We found LC3-positive cells, indicative of autophagy, following inhibition of Nara-H cell growth by Hsp90 blockage. This result suggests that SNX-2112 inhibited cell proliferation and induced autophagy by inhibition of the mTOR pathway in Nara-H cells.

Autophagy is involved in the routine turnover of cell constituents and regulated by mTOR and MAPK signalling pathways (20). The MAPK signalling pathway is critical for tumour formation (21). In this study, MAPK suppression and caspase-dependent apoptosis induction was observed, suggesting that SNX-2112 exerts its antitumor effects in part through apoptosis induced by inhibition of the MAPK pathway.

We demonstrated that SNX-2112 inhibited both mTOR and MAPK signalling pathways. Hsp90 interacts with multiple pathways, including mTOR and MAPK, through its association with relevant proteins, and inhibition of one pathway activates the other (22). Although the mechanism is not fully understood, some functional relationship appears to exist between apoptosis and autophagy (23-25). Our results indicated that suppression of the MAPK pathway induced apoptosis while suppression of the mTOR pathway induced autophagy. These data support the possibility of developing SNX-2112 to treat soft tissue sarcomas.

We previously showed that the Hsp90 inhibitor geldanamycin induced autophagy and apoptosis of osteosarcoma cells and an autophagy inhibitor enhanced apoptosis (26). Similarly, in chronic myeloid leukaemia, an autophagy inhibitor significantly enhanced apoptosis induced by Hsp90 inhibition (27). These reports suggested that autophagy control is important to improve antitumor effects of SNX-2112 because autophagy is a self-defence mechanism employed by cancer cells treated with antitumor agents $(9,11,28)$. However, geldanamycin is too toxic for clinical use. Furthermore, SNX-2112 may suppress 
autophagy induced by MAPK signalling and has a strong antitumor effect without the need to be combined with an autophagy inhibitor. Therefore, SNX-2112 was used in this study.

In conclusion, autophagy and apoptosis were induced in Nara-H cells treated with SNX-2112. Inhibition of phosphorylation of signalling molecules in mTOR and MAPK pathways played a role in these events. Our data support the development of SNX-2112 as a treatment for human soft tissue sarcoma.

\section{References}

1 Reichardt P: High-dose chemotherapy in adult soft tissue sarcoma. Crit Rev Oncol Hematol 41: 157-167, 2002.

2 Spira AI and Ettinger DS: The use of chemotherapy in softtissue sarcomas. Oncologist 7: 348-359, 2002.

3 Zuehlke A and Johnson JL: Hsp90 and co-chaperones twist the functions of diverse client proteins. Biopolymers 93: 211-217, 2010.

4 Isaacs JS, Xu W and Neckers L: Heat shock protein 90 as a molecular target for cancer therapeutics. Cancer Cell 3: 213-217, 2003.

5 Liu KS, Ding WC, Wang SX, Liu Z, Xing GW, Wang Y and Wang YF: The heat shock protein 90 inhibitor SNX-2112 inhibits B16 melanoma cell growth in vitro and in vivo. Oncol Rep 27: 1904-1910, 2012.

6 Amaravadi RK and Thompson CB: The roles of therapy-induced autophagy and necrosis in cancer treatment. Clin Cancer Res 13: 7271-7279, 2007.

7 Palacios C, Martin-Perez R, Lopez-Perez AI, Pandiella A and Lopez-Rivas A: Autophagy inhibition sensitizes multiple myeloma cells to 17-dimethylaminoethylamino-17-demetho-xygeldanamycininduced apoptosis. Leuk Res 34: 1533-1538, 2010.

8 Nakamura O, Hitora T, Yamagami Y, Mori M, Nishimura H, Horie R, Yamaguchi $\mathrm{K}$ and Yamamoto T: The combination of rapamycin and MAPK inhibitors enhances the growth inhibitory effect on Nara-H cells. Int J Mol Med 33: 1491-1497, 2014

9 Kanematsu S, Uehara N, Miki H, Yoshizawa K, Kawanaka A, Yuri $\mathrm{T}$ and Tsubura A: Autophagy inhibition enhances sulforaphane-induced apoptosis in human breast cancer cells Anticancer Res 30: 3381-3390, 2010.

10 Liu D, Yang Y, Liu Q and Wang J: Inhibition of autophagy by 3-MA potentiates cisplatin-induced apoptosis in esophageal squamous cell carcinoma cells. Med Oncol 28: 105-111, 2011.

11 Ren Y, Huang F, Liu Y, Yang Y, Jiang Q and Xu C: Autophagy inhibition through PI3K/Akt increases apoptosis by sodium selenite in NB4 cells. BMB Rep 42: 599-604, 2009.

12 Oliver FJ, de la Rubia G, Rolli V, Ruiz-Ruiz MC, de Murcia G and Murcia JM: Importance of poly(ADP-ribose) polymerase and its cleavage in apoptosis. Lesson from an uncleavable mutant. J Biol Chem 273: 33533-33539, 1998.

13 Li B, Takeda T, Tsuiji K, Wong TF, Tadakawa M, Kondo A, Nagase $S$ and Yaegashi N: Curcumin induces cross-regulation between autophagy and apoptosis in uterine leiomyosarcoma cells. Int J Gynecol Cancer 23: 803-808, 2013.

14 Nakamura O, Hitora T, Akisue T, Kawamoto T, Yamagami Y and Yamamoto T: Inhibition of induced autophagy increases apoptosis of Nara-H cells. Int J Oncol 39: 1545-1552, 2011.

15 Neckers L, Mimnaugh E and Schulte TW: Hsp90 as an anticancer target. Drug Resist Updat 2: 165-172, 1999.
16 Porter JR, Fritz CC and Depew KM: Discovery and development of Hsp90 inhibitors: a promising pathway for cancer therapy. Curr Opin Chem Biol 14: 412-420, 2010.

17 Bachleitner-Hofmann T, Sun MY, Chen CT, Liska D, Zeng Z, Viale A, Olshen, AB, Mittlboeck M, Christensen JG, Rosen N, Solit DB and Weiser MR: Antitumor activity of SNX-2112, a synthetic heat shock protein-90 inhibitor, in MET-amplified tumor cells with or without resistance to selective MET Inhibition. Clin Cancer Res 17: 122-133, 2011.

18 Okawa Y, Hideshima T, Steed P, Vallet S, Hall S, Huang K, Rice J, Barabasz A, Foley B, Ikeda H, Raje N, Kiziltepe T, Yasui H, Enatsu S and Anderson KC: SNX-2112, a selective Hsp90 inhibitor, potently inhibits tumor cell growth, angiogenesis, and osteoclastogenesis in multiple myeloma and other hematologic tumors by abrogating signaling via Akt and ERK. Blood 113: 846-855, 2009.

19 Kim MS, Kuehn HS, Metcalfe DD and Gilfillan AM: Activation and function of the mTORC1 pathway in mast cells. J Immunol 180: 4586-4595, 2008.

20 Kondo Y, Kanzawa T, Sawaya R and Kondo S: The role of autophagy in cancer development and response to therapy. Nat Rev Cancer 5: 726-734, 2005.

21 Sasaki K, Hitora T, Nakamura O, Kono R and Yamamoto T: The role of MAPK pathway in bone and soft tissue tumors. Anticancer Res 31: 549-553, 2011.

22 Saini KS, Loi S, de Azambuja E, Metzger-Filho O, Saini ML, Ignatiadis M, Dancey JE and Piccart-Gebhart MJ: Targeting the $\mathrm{PI} 3 \mathrm{~K} / \mathrm{AKT} / \mathrm{mTOR}$ and Raf/MEK/ERK pathways in the treatment of breast cancer. Cancer Treat Rev 39: 935-946, 2013.

23 Laussmann MA, Passante E, Dussmann H, Rauen JA, Wurstle ML, Delgado ME, Devocelle M, Prehn JH and Rehm M: Proteasome inhibition can induce an autophagy-dependent apical activation of caspase-8. Cell Death Differ 18: 1584-1597, 2011.

24 Liu KS, Liu H, Qi JH, Liu QY, Liu Z, Xia M, Xing GW, Wang SX and Wang YF: SNX-2112, an Hsp90 inhibitor, induces apoptosis and autophagy via degradation of Hsp90 client proteins in human melanoma A-375 cells. Cancer Lett 318: 180-188, 2012.

25 Okuno S: Mammalian target of rapamycin inhibitors in sarcomas. Curr Opin Oncol 18: 360-362, 2006.

26 Mori M, Hitora T, Nakamura O, Yamagami Y, Horie R, Nishimura $\mathrm{H}$ and Yamamoto T: Hsp90 inhibitor induces autophagy and apoptosis in osteosarcoma cells. Int J Oncol 46: 47-54, 2015.

27 He W, Ye X, Huang X, Lel W, You L, Wang L, Chen X and Qian W: Hsp90 inhibitor, BIIB021, induces apoptosis and autophagy by regulating mTOR-Ulk1 pathway in imatinib-sensitive and -resistant chronic myeloid leukemia cells. Int J Oncol 48: 1710-1720, 2016.

28 Horie R, Nakamura O, Yamagami Y, Mori M, Nishimura H, Fukuoka $\mathrm{N}$ and Yamamoto T: Apoptosis and antitumor effects induced by the combination of an mTOR inhibitor and an autophagy inhibitor in human osteosarcoma MG63 cells. Int J Oncol 48: 37-44, 2016.
Received July 20, 2018

Revised July 30, 2018

Accepted July 31, 2018 